\title{
Median Statistics Estimate of the Galactic Rotational Velocity
}

\author{
Tia Camarillo, ${ }^{1 \star}$ Pauline Dredger,${ }^{1} \dagger$ Bharat Ratra ${ }^{1} \ddagger$ \\ ${ }^{1}$ Department of Physics, Kansas State University,116 Cardwell Hall, Manhattan, KS 66506, USA
}

Accepted XXX. Received YYY; in original form ZZZ

\begin{abstract}
We compile a complete collection of 19 recent (since 2000) measurements of $\Theta_{0}$, the rotational velocity of the Milky Way at $R_{0}$ (the radial distance of the Sun from the Galactic center). These measurements use tracers that are believed to more accurately reflect the systematic rotation of the Milky Way. Unlike other recent compilations of $\Theta_{0}$, our collection includes only independent measurements. We find that these 19 measurements are distributed in a mildly non-Gaussian fashion and a median statistics estimate indicates $\Theta_{0}=220 \pm 10 \mathrm{~km} \mathrm{~s}^{-1}$ ( $2 \sigma$ error) as the most reliable summary, at $R_{0}=8.0 \pm 0.3 \mathrm{kpc}(2 \sigma$ error $)$.
\end{abstract}

Key words: (cosmology:) cosmological parameters - Galaxy: fundamental parameters - Galaxy: kinematics and dynamics - Galaxy: structure - methods: data analysis - methods: statistical

\section{INTRODUCTION}

A more accurate model of the Milky Way will improve the accuracy of inter- and extra-galactic measurements. Two constants play a fundamental role in describing the current model of the Milky Way: $R_{0}$ (the radial distance of the Sun to the Galactic center, Sgr $A^{*}$ ) and $\Theta_{0}$ (the rotational velocity of the Milky Way at $R_{0}$ ). Camarillo et al. (2018, hereafter $\mathrm{C} 18$ ) have recently measured $R_{0}$ from a carefully compiled set of independent $R_{0}$ data points. Earlier compilations of data used to estimate $R_{0}$ included non-independent measurements. C18 found that the data set they put together of 28 independent $R_{0}$ results published during 201117 was somewhat non-Gaussian and the results of their median statistics analysis are reasonably well summarized as $R_{0}=8.0 \pm 0.3 \mathrm{kpc}(2 \sigma$ error $)$. After C18 appeared, J. Vallée encouraged us to perform a similar analysis for $\Theta_{0}$.

There have been three recent attempts at measuring $\Theta_{0}$ from compilations of measurements: Vallée (2017, hereafter V17), de Grijs \& Bono (2017, hereafter dGB17), and Rajan \& Desai (2018, hereafter RD18). These analyses use compilations that include non-independent measurements which can significantly affect the results and render them unreliable. In this paper we first put together a collection of 29 independent estimates of $\Theta_{0}$ that have been published in 2000 or later. Of these 29 measurements, 19 correspond to tracers (such as CO and H I gas clouds) that are believed to

\footnotetext{
^ E-mail: tiacamarillo@phys.ksu.edu

$\dagger$ E-mail: pmdredge@ksu.edu

\$ E-mail: ratra@phys.ksu.edu
}

more accurately reflect the systematic rotation of the Milky Way; these are the ones we use to estimate $\Theta_{0}$. We find that this collection of 19 measurements is somewhat nonGaussian so a median statistics analysis (Gott et al. 2001) is needed for a more reliable estimate of $\Theta_{0}$. Using median statistics we find $\Theta_{0}=219.70+6.67+8.77 \mathrm{~km} \mathrm{~s}_{-7}^{+1}(1 \sigma$ and $2 \sigma$ error bars) which for most purposes can be summarized as $\Theta_{0}=220 \pm 7 \pm 10 \mathrm{~km} \mathrm{~s}^{-1}$. Given the extent to which our data compilation is only mildly non-Gaussian, it is likely that undiscovered systematic errors will not significantly change these estimates and $\Theta_{0}=220 \pm 10 \mathrm{~km} \mathrm{~s}^{-1}$ ( $2 \sigma$ error) probably provides the most reliable estimate.

In $\S 2$ we discuss our compilation of recent independent $\Theta_{0}$ measurements and how it differs from those of V17 and dGB17. In $\S 3$ we summarize the central estimates statistics and the tests of Gaussianity. We present and discuss our results $\S 4$. We conclude in $\S 5$.

\section{DATA}

Table 1 lists the $\Theta_{0}$ data we use in our analyses here. These are from measurements published in or after 2000 and we believe this is an exhaustive list of all such independent measurements.

In all cases the angular velocity $\omega_{0 i}=\Theta_{0 i} / R_{0 i}$ was what was measured, so we list $\Theta_{0 i} \pm \sigma_{\Theta_{0 i}}$ and $R_{0 i} \pm \sigma_{R_{0 i}}$ in this table. $\sigma_{\Theta_{0} i}$ and/or $\sigma_{R_{0 i}}$ are not listed in Table 1 if these are not given in the cited reference. In C18 we measure $R_{0} \pm \sigma_{R_{0}}=$ $7.96 \pm 0.17 \mathrm{kpc}$. We use these measurements to compute the 
Table 1. Independent $\Theta_{0}$ measurements since 2000

\begin{tabular}{|c|c|c|c|c|c|}
\hline Radius (kpc) & $\Theta_{0}\left(\mathrm{~km} \mathrm{~s}^{-1}\right)$ & Rescaled $\Theta_{0}^{\text {res }}\left(\mathrm{km} \mathrm{s}^{-1}\right)$ & Reference & Tracer Type & Notes \\
\hline $6.72 \pm 0.39$ & $203.35 \pm 12.00$ & $240.87 \pm 20.59$ & Branham (2014) & Young & Table 3, Hipparcos catalog, 6288 OB stars \\
\hline $7.62 \pm 0.32$ & 205.00 & $214.15 \pm 10.09$ & Battinelli et al. (2013) & Old & Figure 3,4400 carbon stars \\
\hline $7.64 \pm 0.32$ & $217.00 \pm 11.00$ & $226.09 \pm 15.63$ & Bobylev (2013) & Young & Table 3, Cepehids near Sun, UCAC4 \\
\hline $7.97 \pm 0.15$ & $226.80 \pm 4.20$ & $226.52 \pm 7.69$ & McMillan (2017) & Both & In Abstract, from alternative mass model \\
\hline $7.98 \pm 0.79$ & $238.54 \pm 11.66$ & $237.94 \pm 26.76$ & Shen \& Zhang (2010) & Young & From Hipparcos Cepheids \\
\hline 8.00 & $220.80 \pm 13.60$ & $219.70 \pm 14.32$ & Bedin et al. (2003) & Old & $\begin{array}{l}\text { From WFPC } 2 / \text { HST photometry on M4 globular clus- } \\
\text { ter }\end{array}$ \\
\hline $8.00 \pm 0.50$ & $202.70 \pm 24.70$ & $201.69 \pm 27.95$ & Kalirai et al. (2004) & Old & $\begin{array}{l}\text { From HST on M4 globular cluster, independent of } \\
\text { Bedin et al. (2003) }\end{array}$ \\
\hline $8.00 \pm 0.50$ & $236.00 \pm 15.00$ & $234.82 \pm 21.52$ & Reid \& Brunthaler (2004) & Old & From VLBA proper motion around Sgr A* \\
\hline 8.00 & $208.50 \pm 20.00$ & $207.46 \pm 20.39$ & Xue et al. (2008) & Old & Averaged from Table 3 for range $7.5-8.5 \mathrm{kpc}$ \\
\hline 8.00 & $243.50 \pm 13.00$ & $242.28 \pm 13.93$ & Yuan et al. (2008) & Old & From Hipparcos K-M giants \\
\hline 8.00 & 226.84 & $225.71 \pm 4.82$ & Sharma et al. (2011) & Old & $\begin{array}{l}\text { From comparing galaxy model to Hipparcos, Geneva- } \\
\text { Copenhagan survey, and SDSS }\end{array}$ \\
\hline 8.00 & $218.00 \pm 10.00$ & $216.91 \pm 10.98$ & Bovy \& Rix (2013) & Old & Figure 20,16,269 G-type dwarfs, SEGUE \\
\hline $8.00 \pm 0.40$ & $234.00 \pm 14.00$ & $232.83 \pm 18.82$ & Bobylev \& Bajkova (2015) & Young & Data from spectroscopic binaries, in Results section \\
\hline $8.00 \pm 0.40$ & $230.00 \pm 15.00$ & $228.85 \pm 19.43$ & Bobylev \& Bajkova (2015) & Young & Data from Calcium stars distance scale \\
\hline 8.00 & 236.00 & $234.82 \pm 5.02$ & Aumer \& Schönrich (2015) & Young & $\begin{array}{l}\text { Page } 3171 \text {, uses some APOGEE, mostly MW bar } \\
\text { stars }\end{array}$ \\
\hline 8.00 & $227.50 \pm 5.50$ & $226.36 \pm 7.30$ & McGaugh (2016) & Old & $\begin{array}{l}\text { From } \mathrm{CO} \text { and } \mathrm{H} \mathrm{I} \text { clouds, error is average of provided } \\
\text { upper and lower error bars }\end{array}$ \\
\hline 8.00 & $210.00 \pm 10.00$ & $208.95 \pm 10.90$ & Rojas-Arriagada et al. (2016) & Old & Figure 8 , from thin disk stars in Gaia-ESO survey \\
\hline $8.00 \pm 0.40$ & $230.00 \pm 12.00$ & $228.85 \pm 17.24$ & Bobylev \& Bajkova (2016) & Young & In Abstract, from RAVE4 \\
\hline $8.00 \pm 0.20$ & $231.00 \pm 6.00$ & $229.85 \pm 9.63$ & Bobylev (2017) & Young & From Gaia DR1 Cepheids \\
\hline $\begin{array}{l}8.01 \pm 0.44 \\
8.20 \pm 0.70\end{array}$ & $215.00 \pm 24.00$ & $208.71 \pm 29.67$ & Nikiforov $(2000)$ & Old & $\begin{array}{l}\text { From } 5 \text { H I data sets (Nikiforov \& Petrovskaya 1994) } \\
\text { and } 2 \text { CO cloud catalogs (Brand \& Blitz 1993), dif- } \\
\text { fers from McGaugh (2016) }\end{array}$ \\
\hline 8.20 & 238.00 & $231.03 \pm 4.93$ & Portail et al. (2017) & Old & Section 3.4, from red clump stars. \\
\hline $8.24 \pm 0.12$ & $236.50 \pm 7.20$ & $228.46 \pm 9.12$ & Rastorguev et al. (2017) & Old & $\begin{array}{l}\text { In Abstract, error from quadrature addition of error } \\
\text { on } \Theta_{0} \text { and range }\end{array}$ \\
\hline $8.30 \pm 0.25$ & $233.00 \pm 11.35$ & $223.46 \pm 13.66$ & Küpper et al. (2015) & Old & $\begin{array}{l}\text { From Palomar } 5 \text { globular cluster, page } 20 \text {, error is } \\
\text { average of upper and lower provided error bars }\end{array}$ \\
\hline $8.30 \pm 0.20$ & $236.00 \pm 6.00$ & $226.33 \pm 9.29$ & Bobylev et al. (2016) & Young & In Abstract, from MWSC open-clusters catalog \\
\hline $8.34 \pm 0.16$ & $240.00 \pm 6.00$ & $229.06 \pm 8.72$ & Huang et al. (2016) & Old & $\begin{array}{l}\text { From LAMOST/LSS-GAC and SDSS/SEGUE and } \\
\text { SDSS-III/APOGEE, differs from Bovy \& Rix (2013); }\end{array}$ \\
\hline $8.40 \pm 0.40$ & $224.00 \pm 12.50$ & $212.27 \pm 16.22$ & Koposov et al. (2010) & Old & $\begin{array}{l}\text { From SDSS photometry and spectrometry, USNO-B } \\
\text { astrometry, and Calar Alto telescope }\end{array}$ \\
\hline 8.50 & 226.00 & $211.64 \pm 4.52$ & Martínez-Barbosa et al. (2017) & Old & Section 2.4 , from galactic mass modeling \\
\hline
\end{tabular}

recaled

$\Theta_{0 i}^{\mathrm{res}} \pm \sigma_{\Theta_{0 i}}^{\mathrm{res}}=\frac{R_{0} \Theta_{i}}{R_{0 i}}\left(1 \pm \sqrt{\left(\frac{\sigma_{R_{0}}}{R_{0}}\right)^{2}+\left(\frac{\sigma_{\Theta_{0 i}}}{\Theta_{0 i}}\right)^{2}+\left(\frac{\sigma_{R_{0 i}}}{R_{0 i}}\right)^{2}}\right)$

and list these in column 3 of Table $1 .^{1}$

It has been known for quite a while now that $\Theta_{0}$ measured using different tracers differ (Roman 1950, 1952; Avedisova 2005; Yuan et al. 2008; dGB17, and references therein). We categorize the measurements listed in Table 1 into either Old or Young (one publication uses both types of tracers and is listed as Both). Old tracers include CO and $\mathrm{H}$ I gas clouds and are thought to better reflect the systematic rotation of the Galaxy while Young tracers such as Cepheids are believed to have velocities that are contaminated by "peculiar" motions. In Table 1 we have 18+1 Old measurements and $10+1$ Young ones.

Unlike the measurements listed in Table 1, the collections compiled by V17 and dGB17 include non-independent data points. In their analyses dGB17 and RD18 consider different subsets of data, based on tracer type and/or year of publication, but like V17 they also do not study a compilation of independent measurements. This lack of independence can bias results. Here we have invested significant effort in compiling a collection of independent $\Theta_{0}$ measurements published during 2000-2017.

1 More properly one would use the rescaled angular velocities in the analysis and then convert the resulting angular velocity central value to a linear velocity central value. However, the uncertainty on $R_{0}$ is small and so results from the two different approaches will only differ slightly.

\section{METHODS OF ANALYSIS}

It is expected that a large enough data set of $N$ independent measurements will follow a Gaussian distribution, however it is not unheard of for an astronomical parameter to not obey a Gaussian distribution. ${ }^{2}$ Here we study the data compilation of Table 1 (and two subcompilations) to examine if it is non-Gaussian or not. If it is non-Gaussian this could be caused by improperly estimated errors.

To estimate the Gaussianity of a data collection we need to use a central estimate of the data. We consider two here, the median central estimate and the weighted mean central estimate.

Median statistics does not use information of the error on a measurement at all and the true median of a data set can be found independent of any of the individual measurements errors. The estimated median will have a larger uncertainty than that of a weighted central estimate statistic that makes use of error information. We use the median statistics technique developed by Gott et al. (2001). The median is defined as the value with $50 \%$ probability of finding another value above and below it. Gott et al. (2001) show that for a dataset of $i=1,2, \ldots, N$ independent values, $\Theta_{i}$,

2 Perhaps the most famous example is the Hubble constant (Chen et al. 2003; Chen \& Ratra 2011a). For other examples in astronomy, cosmology, and physics see Farooq et al. (2013); Crandall et al. (2015); Farooq et al. (2017); Bailey (2017); Zhang (2017), and references therein. Significant effort is devoted to testing for intrinsic non-Gaussianity in physical systems (e.g. Park et al. 2001; Planck Collaboration 2016), as opposed to measurement induced non-Gaussianity, since Gaussianity is usually assumed in parameter estimation (e.g. Samushia et al. 2007; Chen \& Ratra 2011b; Ooba et al. 2017). 
the probability of the median being between $\Theta_{i}$ and $\Theta_{i+1}$ is given by the binomial distribution

$P=\frac{2^{-N} N !}{i !(N-1) !}$.

The $1 \sigma$ error about the median is then defined by the range about it such that $68.27 \%$ of the probability is included. This can be extended to finding the $2 \sigma$ error about the median, where instead $95.45 \%$ of the probability would be enclosed. We refer to the median of the Galactic rotational velocity at $R_{0}$ as $\Theta_{0}^{\text {med }}$.

The weighted mean comes with the benefit of additional information in the errors, at the potential expense of including inaccurate uncertainties (Podariu et al. 2001). The weighted mean of the Galactic rotational velocity is

$\Theta_{0}^{\mathrm{wm}}=\frac{\sum_{i=1}^{N} \Theta_{0 i} / \sigma_{\Theta_{0} i}{ }^{2}}{\sum_{i=1}^{N} 1 / \sigma_{\Theta_{0} i}{ }^{2}}$,

where $\Theta_{0 i}$ and $\sigma_{\Theta_{0 i}}$ are the rotational velocities and errors. The weighted mean standard deviation is

$\sigma_{\Theta_{0}}^{\mathrm{wm}}=\frac{1}{\sqrt{\sum_{i=1}^{N} 1 / \sigma_{\Theta_{0}}^{2}}}$.

The next step in analyzing the data is to construct error distributions of the data based on the chosen central estimate. For a central estimate $\Theta_{0}^{\mathrm{CE}}$ independent of the data $\Theta_{0 i}$, the number of standard deviations that each value deviates from the central estimate is

$N_{\sigma_{\Theta_{0} i}}=\frac{\Theta_{0 i}-\Theta_{0}^{\mathrm{CE}}}{\sqrt{\operatorname{Var}\left(\Theta_{0 i}-\Theta_{0}^{\mathrm{CE}}\right)}}$

where $\operatorname{Var}\left(\Theta_{0 i}-\Theta_{0}^{\mathrm{CE}}\right)$ is the variance between the independent measurement, $\Theta_{0 i}$ and the central estimate, $\Theta_{0}^{\mathrm{CE}}$.

For median statistics when the central estimate is assumed to not be directly correlated with the data itself we have

$N_{\sigma_{\Theta_{0 i}}}^{\mathrm{med}}=\frac{\Theta_{0 i}-\Theta_{0}^{\mathrm{med}}}{\sqrt{\left(\sigma_{\Theta_{0 i}}\right)^{2}+\left(\sigma_{\Theta_{0 i}}^{\mathrm{med}}\right)^{2}}}$.

For Gaussianly distributed measurements and the weighted mean central estimate estimated from the data (and so correlated with the data) we instead have (see the Appendix of $\mathrm{C} 18)^{3}$

$N_{\sigma_{\Theta_{0 i}}}^{\mathrm{wm}}=\frac{\Theta_{0 i}-\Theta_{0}^{\mathrm{wm}}}{\sqrt{\left(\sigma_{\Theta_{0 i}}\right)^{2}-\left(\sigma_{\Theta_{0 i}}^{\mathrm{wm}}\right)^{2}}}$.

The two error distributions, $N_{\sigma_{\Theta_{0}}}^{\mathrm{med}}$ and $N_{\sigma_{\Theta_{0}}}^{\mathrm{wm}}$, can be analyzed with a non-parametric Kolmogorov-Smirnov (KS) test.

We compare these error distributions to a few standard functional forms (e.g. Crandall \& Ratra 2015; C18). The four probability distribution functions (PDFs) we consider here are the standard Gaussian PDF, the Cauchy

3 An analogous equation for median statistics, for the case when the median is estimated from the data and so is correlated with the data, is not yet known.
(Lorentzian) PDF, the Student's $t$ PDF, and the Laplacian (double exponential) PDF. The KS test allows us to quantify the level of deviation from Gaussianity of the error distributions by using the outputs of this test, the $D$-statistic and the $p$-value. For a 95\% confidence level in the probability that we cannot reject a specific PDF as describing the data, we look for two requirements for a data set of 29 measurements: $D \rightarrow 0$ but $\leq 0.246$, and $p \rightarrow 1$. For the Old tracers (19 measurements) we require almost the same: $D \rightarrow 0$ but $\leq 0.301$, and $p \rightarrow 1$, and for Young tracers (11 measurements) we require $D \rightarrow 0$ but $\leq 0.391$, and $p \rightarrow 1.4$

The standard Gaussian PDF is

$P(|\mathbf{X}|)=\frac{1}{\sqrt{2 \pi}} \exp \left(-|\mathbf{X}|^{2} / 2\right)$,

and is characterized by $1 \sigma(2 \sigma)$, or $68.27 \%(95.45 \%)$, of the data falling within $|X| \leq 1(|X| \leq 2)$.

The Cauchy PDF has higher probability in the tails of the curve, and $1 \sigma(2 \sigma)$, or $68.27 \%$ (95.45\%), of the data falls within $|X| \leq 1.8(|X| \leq 14)$. The Cauchy PDF is

$P(|\mathbf{X}|)=\frac{1}{\pi} \frac{1}{1+|\mathbf{X}|^{2}}$.

The Student's $t$ distribution also has widened tails, and involves an additional parameter $n$. A Student's $t$ of $n=1$ is the Cauchy PDF, and as $n \rightarrow \infty$, it becomes the Gaussian. The PDF is

$P(|\mathbf{X}|)=\frac{\Gamma[(n+1) / 2]}{\sqrt{\pi n} \Gamma(n / 2)} \frac{1}{\left(1+|\mathbf{X}|^{2} / n\right)^{(n+1) / 2}}$

and the limits for $1 \sigma(2 \sigma)$, or $68.27 \%$ (95.45\%), of the data varies with $n$. Here $\Gamma$ is the gamma function and the addition of $n$ (positive integer parameter) decreases the total degrees of freedom by one.

The last PDF we consider is the Laplacian

$P(|\mathbf{X}|)=\frac{1}{2} \exp (-|\mathbf{X}|)$.

It is characterized by $1 \sigma(2 \sigma)$, or $68.27 \%(95.45 \%)$, of the data falling within $|X| \leq 1.2(|X| \leq 3.1)$. This results in a more sharply peaked distribution than either a Cauchy or a Student's $t$.

In eqs. (8), (9), (10), and (11) the value $|\mathbf{X}|=\left|\Theta_{0} / S\right|$. $S$ is a scale factor width for each distribution $(S=1$ for a Gaussian PDF represents the standard normal distribution). We allow $S$ to vary in small increments of 0.001 from $S=$ 0 to 5 , and for the Student's $t$ we do this for every value of $n$ from $n=2$ to 100 . The KS test, being non-parametric, makes no assumptions about the data.

\section{RESULTS}

We provide in Table 2 the central estimate statistics for the data listed in column 3 of Table 1 . In Table 2, column 2 shows the median (with $1 \sigma$ and $2 \sigma$ error ranges) and weighted mean results for all 29 values. Column 3 shows the results of only analyzing the 18 Old tracer references, plus
4 See C18 and Appendix 3 of O'Connor \& Kleyner (2012) for more detailed discussion of the outputs of the KS tests and the critical values for below which $D$ must fall. 
the mixed tracer type of McMillan (2017). Column 4 shows the results of the 10 Young tracer types, plus the mixed tracer type as well.

While Table 3 shows the highest probabilities for Young tracer types, with all probabilities $p \geq 0.99$, the scale factors for all these PDFs are very non-Gaussian with all of them having $1 \sigma$ ranges requiring $|X| \leq 0.5$. The All tracers compilation is also fairly non-Gaussian.

For the Old tracers collection with the median as the central estimate, $p=0.83$ while $S=1.08$ for the Gaussian $\mathrm{PDF}$, indicating not unreasonable consistency with Gaussianity. This is also supported by the weighted mean result for the Gaussian PDF. Together these results indicate that the weighted mean summary for $\Theta_{0}$ is less appropriate than our median statistics one of $\Theta_{0}=219.70+6.67+8.77 \mathrm{~km} \mathrm{~s}_{-7}^{+1}$ ( $1 \sigma$ and $2 \sigma$ errors), which for most purposes can be taken to be $\Theta_{0}=220 \pm 7 \pm 10 \mathrm{~km} \mathrm{~s}^{-1}$. In summary, for practical purposes, we find at $1 \sigma$ :

$$
\begin{aligned}
& \Theta_{0}=220 \pm 7 \mathrm{~km} \mathrm{~s}^{-1} \\
& R_{0}=7.96 \pm 0.17 \mathrm{kpc} \\
& \omega_{0}=\Theta_{0} / R_{0}=27.6 \pm 1.1 \mathrm{~km} \mathrm{~s}^{-1} \mathrm{kpc}^{-1}
\end{aligned}
$$

where the angular speed $\omega_{0}$ error is determined by adding the fractional uncertainties of $\Theta_{0}$ and $R_{0}$ in quadrature.

Table 1 of V17 lists 28 measurements of $\Theta_{0}$ from mid2012 to 2017 . V17 arrives at a $\Theta_{0}$ close to $230 \mathrm{~km} \mathrm{~s}^{-1}$ : median value $\Theta_{0}^{\text {med }}=232 \mathrm{~km} \mathrm{~s}^{-1}$, weighted mean value $\Theta_{0}^{\mathrm{wm}}=228 \pm 2$ $\mathrm{km} \mathrm{s}^{-1}$, and an arithmetic mean value $\Theta_{0}^{\text {mean }}=229 \pm 3 \mathrm{~km}$ $\mathrm{s}^{-1}$. He recommends the set of Galactic constants:

$$
\begin{aligned}
& \Theta_{0}=230 \pm 3 \mathrm{~km} \mathrm{~s}^{-1} \\
& R_{0}=8.0 \pm 0.2 \mathrm{kpc} \\
& \omega_{0}=\Theta_{0} / R_{0}=29 \pm 1 \mathrm{~km} \mathrm{~s}^{-1} \mathrm{kpc}^{-1}
\end{aligned}
$$

We emphasize that several of the V17 Table 1 data are repeats of prior publications, big offenders being masers, OB stars, and Cepheids. Less than half of V17 Table 1 measurements are included in our list of independent measurements. V17 also does not distinguish between Old and Young tracer measurements of $\Theta_{0}$. These are probably why the V17 $\Theta_{0}$ differs from our estimate.

dGB17 on the other hand do note that Old tracers provide a better estimate of $\Theta_{0}$ and their recommended set of Galactic constants are (when their statistical and systematic errors are added in quadrature):

$$
\begin{aligned}
& \Theta_{0}=225 \pm 10 \mathrm{~km} \mathrm{~s}^{-1} \\
& R_{0}=8.3 \pm 0.4 \mathrm{kpc} \\
& \omega_{0}=\Theta_{0} / R_{0}=27.1 \pm 1.8 \mathrm{~km} \mathrm{~s}^{-1} \mathrm{kpc}^{-1} .
\end{aligned}
$$

While their Old tracers compilation includes nonindependent data points, dGB17 add on rather large undiscovered systematic errors and so their results are not inconsistent with our results. We note, in particular, as described in $\mathrm{C} 18$, that their estimate of $R_{0}$ is based on a very small set of data points (that are also not all independent). We emphasize that from our analysis of the Gaussianity of our $R_{0}$ and $\Theta_{0}$ compilations, here and in $\mathrm{C} 18$, we do not see strong evidence for large undiscovered systematic errors that dGB17 advocate for.

RD18 use 139 Galactic rotation speed values, 137 of which are from the online database of dGB17. Included are a number of non-independent measurements. For both median and weighted mean statistics they use the error distribution form of eq. (6) and analyze the full collection of data as well as various subsets. RD18 were the first to realize that the
dGB17 $\Theta_{0}$ data (and subsets) was non-Gaussian, but as they didn't discard non-independent measurements (as we have done) they found the data to be more non-Gaussian than we do. From a median statistics analysis of the full data set they recommend:

$$
\begin{aligned}
& \Theta_{0}=219.65 \mathrm{~km} \mathrm{~s}^{-1} \\
& R_{0}=8.3 \mathrm{kpc} \\
& \omega_{0}=\Theta_{0} / R_{0}=26.46 \mathrm{~km} \mathrm{~s}^{-1} \mathrm{kpc}^{-1} .
\end{aligned}
$$

They do not derive an $R_{0}$ value, instead they use that estimated by dGB17. They also do not estimate an error for $\Theta_{0}$.

\section{CONCLUSION}

The data listed in Table 1 is the first compilation of independent $\Theta_{0}$ measurements published during 2000-2017. Given the mild non-Gaussianity of the Old tracer measurements, we favor a median statistics value of $\Theta_{0}=219.70_{-7.43}^{+6.67+8.77}$, $\mathrm{km} \mathrm{s}^{-1}$ ( $1 \sigma$ and $2 \sigma$ errors). For most purposes this can be summarized as $\Theta_{0}=220 \pm 7 \pm 10 \mathrm{~km} \mathrm{~s}^{-1}$. Given that the measured non-Gaussianity is mild, we believe most current $\Theta_{0}$ error bars are reasonable and that at present there is no strong evidence for large undiscovered systematic errors. In summary our recommended set of Galactic constants, with $1 \sigma$ error bars,

$$
\begin{aligned}
& \Theta_{0}=220 \pm 7 \mathrm{~km} \mathrm{~s}^{-1} \\
& R_{0}=7.96 \pm 0.17 \mathrm{kpc} \\
& \omega_{0}=\Theta_{0} / R_{0}=27.6 \pm 1.1 \mathrm{~km} \mathrm{~s}^{-1} \mathrm{kpc}^{-1}
\end{aligned}
$$

are probably the most reliable.

\section{ACKNOWLEDGEMENTS}

We thank A. Quillen and J. Vallée. This research was supported in part by DOE grant DE-SC0011840.

\section{REFERENCES}

Aumer M., Schönrich R., 2015, MNRAS, 454, 3166

Avedisova V. S., 2005, Astronomy Reports, 49, 435

Bailey D. C., 2017, Royal Society Open Science, 4, 160600

Battinelli P., Demers S., Rossi C., Gigoyan K. S., 2013, Astrophysics, 56, 68

Bedin L. R., Piotto G., King I. R., Anderson J., 2003, AJ, 126, 247

Bobylev V. V., 2013, Astronomy Letters, 39, 753

Bobylev V. V., 2017, Astronomy Letters, 43, 152

Bobylev V. V., Bajkova A. T., 2015, Astronomy Letters, 41, 473

Bobylev V. V., Bajkova A. T., 2016, Astronomy Letters, 42, 90

Bobylev V. V., Bajkova A. T., 2017, Astronomy Letters, 43, 159

Bobylev V. V., Bajkova A. T., Shirokova K. S., 2016, Astronomy Letters, 42, 721

Bovy J., Rix H.-W., 2013, The Astrophysical Journal, 779, 115

Brand J., Blitz L., 1993, A\&A, 275, 67

Branham R. L., 2014, Ap\&SS, 353, 179

Camarillo T., Mathur V., Mitchell T., Ratra B., 2018, PASP, 130, 024101

Chen G., Ratra B., 2011a, PASP, 123, 1127

Chen Y., Ratra B., 2011b, Physics Letters B, 703, 406

Chen G., Gott III J. R., Ratra B., 2003, PASP, 115, 1269

Crandall S., Ratra B., 2015, ApJ, 815, 87

Crandall S., Houston S., Ratra B., 2015,

Modern Physics Letters A, 30, 1550123

de Grijs R., Bono G., 2017, ApJS, 232, 22 
Table 2. Central estimates of rescaled $\Theta_{0}$ data (in $\mathrm{km} \mathrm{s}^{-1}$ )

\begin{tabular}{lccc}
\hline Statistic & All Tracers & Old Tracers & Young Tracers \\
\hline Median & $226.35+2.12+2.50$ & $219.70+6.67+8.77-10.75$ & $228.85+3.98+9.09$ \\
$1 \sigma$ range & $223.46-228.46$ & $212.27-226.36$ & $226.52-232.83$ \\
$2 \sigma$ range & $217.91-228.85$ & $208.95-228.46$ & $226.09-237.94$ \\
\hline Weighted Mean & $224.36 \pm 1.67$ & $222.01 \pm 1.99$ & $230.05 \pm 3.09$ \\
$1 \sigma$ range & $222.69-226.03$ & $220.02-224.00$ & $226.95-233.14$ \\
\hline
\end{tabular}

Table 3. $N_{\sigma}$ KS test results for rescaled $\Theta_{0}$

\begin{tabular}{|c|c|c|c|c|c|c|}
\hline \multirow[b]{2}{*}{ Type } & \multicolumn{3}{|c|}{$N_{\sigma_{\Theta_{0}}}^{\mathrm{med}}$} & \multicolumn{3}{|c|}{$N_{\sigma_{\Theta_{0}}}^{\mathrm{wm}}$} \\
\hline & PDF & $p^{\mathrm{a}}$ & $S^{\mathrm{b}}$ & PDF & $p^{\mathrm{a}}$ & $S^{\mathrm{b}}$ \\
\hline \multirow[t]{4}{*}{ All } & Gaussian & 0.49 & 0.75 & Gaussian & 0.20 & 0.73 \\
\hline & Cauchy & 0.70 & 0.46 & Cauchy & 0.34 & 0.45 \\
\hline & $n=2$ Student's $t$ & 0.59 & 0.59 & $n=2$ Student's $t$ & 0.26 & 0.58 \\
\hline & Laplace & 0.62 & 0.69 & Laplace & 0.29 & 0.68 \\
\hline \multirow[t]{4}{*}{ Old } & Gaussian & $0.83^{\mathrm{c}}$ & 1.08 & Gaussian & 0.78 & 1.26 \\
\hline & Cauchy & 0.71 & 0.70 & Cauchy & 0.81 & 0.89 \\
\hline & $n=36$ Student's $t$ & $0.83^{\mathrm{d}}$ & 1.07 & $n=2$ Student's $t$ & 0.80 & 1.06 \\
\hline & Laplace & 0.76 & 1.04 & Laplace & 0.81 & 1.27 \\
\hline \multirow[t]{4}{*}{ Young } & Gaussian & 0.99 & 0.35 & Gaussian & 0.99 & 0.38 \\
\hline & Cauchy & 0.99 & 0.18 & Cauchy & 0.99 & 0.20 \\
\hline & $n=2$ Student's $t$ & 0.99 & 0.23 & $n=2$ Student's $t$ & 0.99 & 0.26 \\
\hline & Laplace & 0.99 & 0.27 & Laplace & 0.99 & 0.30 \\
\hline
\end{tabular}

a The probability ( $p$-value) that the input data doesn't not come from the PDF.

b The scale factor $S$ that maximizes $p$.

${ }^{\mathrm{c}}$ More precisely, $p=0.82817$.

d More precisely, $p=0.82811$.

Farooq O., Crandall S., Ratra B., 2013, Physics Letters B, 726, 72 Farooq O., Ranjeet Madiyar F., Crandall S., Ratra B., 2017, ApJ, 835,26

Gott III J. R., Vogeley M. S., Podariu S., Ratra B., 2001, ApJ, 549,1

Huang Y., et al., 2016, MNRAS, 463, 2623

Kalirai J. S., et al., 2004, ApJ, 601, 277

Koposov S. E., Rix H.-W., Hogg D. W., 2010, ApJ, 712, 260

Küpper A. H. W., Balbinot E., Bonaca A., Johnston K. V., Hogg D. W., Kroupa P., Santiago B. X., 2015, ApJ, 803, 80

Martínez-Barbosa C. A., Jílková L., Portegies Zwart S., Brown A. G. A., 2017, MNRAS, 464, 2290

McGaugh S. S., 2016, ApJ, 816, 42

McMillan P. J., 2017, MNRAS, 465, 76

Nikiforov I. I., 2000, in Valtonen M. J., Flynn C., eds, Astronomical Society of the Pacific Conference Series Vol. 209, IAU Colloq. 174: Small Galaxy Groups. p. 403

Nikiforov I. I., Petrovskaya I. V., 1994, Azh, 71, 725

O'Connor P. D. T., Kleyner A., 2012, Practical Reliability Engineering, 5 edn. Wiley

Ooba J., Ratra B., Sugiyama N., 2017, preprint, (arXiv:1707.03452)

Park C.-G., Park C., Ratra B., Tegmark M., 2001, ApJ, 556, 582

Planck Collaboration 2016, A\&A, 594, A17

Podariu S., Souradeep T., Gott III J. R., Ratra B., Vogeley M. S., 2001, ApJ, 559, 9

Portail M., Gerhard O., Wegg C., Ness M., 2017, MNRAS, 465,1621

Rajan A., Desai S., 2018, European Physical Journal Plus, 133,107

Rastorguev A. S., Utkin N. D., Zabolotskikh M. V., Dambis A. K., Bajkova A. T., Bobylev V. V., 2017, Astrophysical Bulletin,
72,122

Reid M. J., Brunthaler A., 2004, ApJ, 616, 872

Rojas-Arriagada A., et al., 2016, A\&A, 586, A39

Roman N. G., 1950, ApJ, 112, 554

Roman N. G., 1952, ApJ, 116, 122

Samushia L., Chen G., Ratra B., 2007, preprint, (arXiv:0706.1963)

Sharma S., Bland-Hawthorn J., Johnston K. V., Binney J., 2011, ApJ, 730, 3

Shen M., Zhang H., 2010, Chinese Astron. Astrophys., 34, 89

Vallée J. P., 2017, Ap\&SS, 362, 79

Xue X. X., et al., 2008, ApJ, 684, 1143

Yuan F.-T., Zhu Z., Kong D.-L., 2008, Chinese J. Astron. Astrophys., 8, 714

Zhang J., 2017, MNRAS, 468, 5014

This paper has been typeset from a $\mathrm{T}_{\mathrm{EX}} / \mathrm{LAT}_{\mathrm{EX}}$ file prepared by the author. 